Jochen Koenigsmann*

\title{
The regular inverse Galois problem over non-large fields
}

Received October 18, 2002 and in revised form November 25, 2003

\begin{abstract}
By a celebrated theorem of Harbater and Pop, the regular inverse Galois problem is solvable over any field containing a large field. Using this and the Mordell conjecture for function fields, we construct the first example of a field $K$ over which the regular inverse Galois problem can be shown to be solvable, but such that $K$ does not contain a large field. The paper is complemented by model-theoretic observations on the diophantine nature of the regular inverse Galois problem.
\end{abstract}

Keywords. Inverse Galois problem, embedding problems, large fields, Mordell conjecture for function fields, diophantine theory of fields

\section{Introduction}

The regular inverse Galois problem over a field $K$ is the problem to realize any finite group $G$ regularly over $K$, i.e. to find a Galois extension $L / K(t)$ with group $G$, where $t$ is an indeterminate over $K$ and $L / K$ is regular. A fundamental theorem of Harbater-Pop says that the regular inverse Galois problem is solvable over any large field $K]^{1}([\mathrm{H}],[\mathrm{P}]$, see also $[\mathrm{HJ}]$ and $[\mathrm{CT}])$. This implies that the regular inverse Galois problem is solvable for any field $K$ containing a large field $k$ : If $t$ is transcendental over $K$ and $l / k(t)$ is a Galois extension with group $G$, regular over $k$, then $L:=K l / K(t)$ is a Galois extension with group $G$, regular over $K$.

In this note we shall construct a field $K$ over which the regular inverse Galois problem is solvable but which does not contain any large field (Theorem 2.1). It is based on a reformulation of the theorem of Harbater-Pop mentioned above (Theorem 1.1) and on the Mordell Conjecture over function fields $([\mathrm{M}]$ in characteristic 0 with correction in $[\mathrm{C}]$, and $[\mathrm{G}]$ and $[\mathrm{S}]$ in arbitrary characteristic).

The last part of the paper contains model-theoretic considerations on the diophantine nature of the regular inverse Galois problem. More precisely, we show that for each finite

*Heisenberg-Stipendiat der Deutschen Forschungsgemeinschaft (KO 1962/1-2).

J. Koenigsmann: Mathematisches Institut der Universität Freiburg, Abteilung mathematische Logik und Grundlagen der Mathematik, Eckerstr. 1, 79104 Freiburg, Germany; e-mail: Jochen.Koenigsmann@unibas.ch

Mathematics Subject Classification (2000): Primary 12F12; Secondary 12E30, 12L12 14G05

1 Recall that a field $K$ is large if $K$ is existentially closed in $K((x))$, or, equivalently, if any smooth curve over $K$ with one $K$-rational point has infinitely many $K$-rational points. 
group $G$ there is a sequence $(\operatorname{REG}(G, n))_{n \in \mathbb{N}}$ of first-order existential statements in the language $\{+, \cdot, 0,1\}$ of rings such that for any $n, \operatorname{REG}(G, n)$ implies $\operatorname{REG}(G, n+1)$, and such that for any field $K$,

$$
G \text { regularly realizable over } K \Leftrightarrow K \models \operatorname{REG}(G, n) \text { for some } n \in \mathbb{N} \text {. }
$$

The formulas REG $(G, n)$ may be considered as a simple variant of the Hurwitz spaces introduced in [FV]. Lacking their rich structure, however, our formulas have the advantage of being easily and effectively constructed (using only quantifier elimination for algebraically closed fields) and working simultaneously in all characteristics.

We illustrate this point of view by a slight generalization of the theorem of HarbaterPop (Observation 3.3 replacing the condition that $K$ be large by the weaker condition that $\sharp \mathcal{C}(K)=\infty$ for any smooth curve $\mathcal{C}$ defined over the prime field $k \subseteq K$ (so $k=\mathbb{Q}$ or $\left.\mathbb{F}_{p}\right)$ with $\mathcal{C}(k) \neq \emptyset$. We should mention, however, that this generalization could also be achieved without model theory by using the classical Bertini-Noether specialization argument as used in the proof of Theorem B in $[\overline{\mathrm{P}}]$ or as described in Section 4.2 of [DD].

\section{Reformulating Harbater-Pop}

The theorem of Harbater-Pop mentioned in the Introduction, in fact, says more than regular realizability of all finite groups over large fields. More generally, it asserts regular proper solvability of all finite split embedding problems over such fields.

Recall that an embedding problem for a field $K$ is a pair of epimorphisms $\mathbf{E P}_{K}=$ $\left(\alpha_{K}: G_{K} \rightarrow A, \beta: B \rightarrow A\right)$ of profinite groups, where $G_{K}:=\operatorname{Gal}\left(K^{s} / K\right)$ is the absolute Galois group of $K$. It is finite if $B$ is finite, and split if $\beta$ splits. A solution of $\mathbf{E P}_{K}$ is a homomorphism $\gamma: G_{K} \rightarrow B$ with $\alpha_{K}=\beta \circ \gamma$. When $\gamma$ is onto, it is a proper solution. Let $L$ be the fixed field of the kernel of $\alpha_{K}$, i.e. $L / K$ is a Galois extension with group $A$. If $F / K$ is a field extension linearly disjoint from $L$ over $K$ (e.g. $F / K$ regular) then $F L / F$ is again Galois with group $A$, and $\mathbf{E} \mathbf{P}_{K}$ induces the lifted embedding problem $\mathbf{E P}_{F}=\left(\alpha_{F}:=\alpha_{K} \circ \operatorname{res}_{F / K}, \beta\right)$, where $\operatorname{res}_{F / K}$ is the restriction homomorphism $G_{F} \rightarrow G_{K}$. A regular (proper) solution of $E P_{K}$ is a (proper) solution $\gamma$ of the lifted embedding problem $\mathbf{E} \mathbf{P}_{K(t)}$, where $t$ is transcendental over $K$, and the fixed field of $\operatorname{ker} \gamma$ is a regular extension of the fixed field of $\operatorname{ker} \alpha_{K(t)}=L(t)$. If $F / K$ is linearly disjoint from $L$ over $K$ then any regular solution of $\mathbf{E P} \mathbf{P}_{K}$ lifts to a regular solution of $\mathbf{E} \mathbf{P}_{F}$.

The following version of the theorem of Harbater-Pop is certainly not new to the experts. It follows the lines of [DD, Section 4.2], where the case of regular realizability of a given finite group $B$ (i.e. embedding problems with $A=\{1\}$ ) is treated. It presents the theorem as a general field-theoretic fact, no longer restricted to a special class of fields.

Theorem 1.1. Let $K$ be a field and let $\mathbf{E} \mathbf{P}_{K}=\left(\alpha_{K}: G_{K} \rightarrow A, \beta: B \rightarrow A\right)$ be $a$ finite split embedding problem for $K$. Then there is a function field $F / K$ in one variable with a $K$-rational place such that the lifted embedding problem $\mathbf{E P}_{F}$ has a proper regular solution over $F$. 
Proof. Let $L=$ Fix $\operatorname{ker}\left(\alpha_{K}\right)$ be the fixed field of the kernel of $\alpha_{K}$, so $L$ is a finite Galois extension of $K$ with group $A$. Let $x$ be an indeterminate over $K$ and let $F^{\prime}$ be a henselization of $K(x)$ w.r.t. the $x$-adic valuation on $K(x)$, i.e. $F^{\prime}=K((x)) \cap K(x)^{\mathrm{s}}$, where $K(x)^{\mathrm{s}}$ denotes a separable algebraic closure of $K(x)$. Then $F^{\prime} / K$ is a regular extension of transcendence degree 1 and $F^{\prime}$ is large, having a non-trivial henselian valuation. By Harbater-Pop, the lifted embedding problem $\mathbf{E P}{F_{F}}$ has then a regular solution over $F^{\prime}$, i.e. there is a Galois extension $E^{\prime} / F^{\prime}(t)$ ( $t$ transcendental over $\left.F^{\prime}\right)$ containing $F^{\prime} L$ and regular over $F^{\prime} L$ such that $\operatorname{Gal}\left(E^{\prime} / F^{\prime}(t)\right)=B$ and $\beta$ is the restriction map

$$
\beta: \operatorname{Gal}\left(E^{\prime} / F^{\prime}(t)\right) \rightarrow \operatorname{Gal}\left(F^{\prime} L / F^{\prime}\right) \cong \operatorname{Gal}(L / K)=A .
$$

Now let $F$ be a finite subextension of $F^{\prime} / K(x)$ such that $E^{\prime} / F^{\prime}(t)$ is defined over $F$, i.e. there is a Galois extension $E / F(t)$ containing $L$ with $E^{\prime}=F^{\prime} E$ and $\operatorname{Gal}\left(E^{\prime} / F^{\prime}(t)\right)$ $\cong \mathrm{Gal}(E / F(t))$. As subfield of $K((x)), F$ is regular over $K$ with a $K$-rational place, and $E / F(t)$ provides a proper regular solution of the lifted embedding problem $\mathbf{E P}_{F}$.

Remark 1.2. The above theorem is, indeed, a reformulation of the theorem of HarbaterPop, as the latter is also easily deduced from the former.

Proof. Let $K$ be a large field and let $\mathbf{E P}_{K}$ be a finite split embedding problem over $K$. Then, by Theorem 1.1. $\mathbf{E P}_{F}$ has a proper regular solution over $F$ for some function field $F / K$ in one variable admitting a $K$-rational place. By Hensel's Lemma, $F$ can be embedded in $K((x))$, where $x \in F$ is a uniformizing element of this place. Since $K((x)) / F$ is linearly disjoint from $F L$ over $F$, we can lift the embedding problem $\mathbf{E} \mathbf{P}_{F}$ and its regular solution over $F$ to $K((x))$.

As $K$ is large, it is existentially closed in $K((x))$. To go down to $K$ we can, therefore, apply [ [P Lemma 1.5], or, similar to the proof of Theorem 3.1, we can write down directly the existential formula $\phi$ with parameters from $K$ (defining the extension $L / K$ ) such that $\phi$ is satisfied by the coefficients from $K((x))$ occurring in explicit polynomials describing a regular solution of $\mathbf{E P}_{K((x))}$ and such that any extension $F^{\prime} / K$ linearly disjoint from $L$ over $K$ and satisfying $\phi$ allows a regular solution of $\mathbf{E} \mathbf{P}_{F^{\prime}}$. As $K$ is existentially closed in $K((x))$, this is, in particular, the case for $F^{\prime}=K$.

Remark 1.3. Note that the function field $F$ in Theorem 1.1 can be chosen to be a rational function field iff $\mathbf{E P} \mathbf{P}_{K}$ is regularly solvable over $K$.

Proof. This is because, by Bertini-Noether, regular solvability of $\mathbf{E} \mathbf{P}_{K}$ and of $\mathbf{E P}_{K(x)}$ is the same.

Remark 1.4. For regular realizability of a finite group $B$ (i.e. embedding problems with $A=\{1\}$ ), the conclusion of Theorem 1.1 without the existence of $K$-rational places on $F$ follows already from the existence of absolutely irreducible components in Hurwitz spaces, at least in characteristic 0 (cf. Part 1 of the proof of Corollary 2 in [FV p. 785]). One has to use standard techniques for the descent from a higher dimensional function field to the dimension 1 case though. 


\section{New fields}

So far the only examples of fields over which the regular inverse Galois problem could be shown to be solvable were fields containing a large field. We shall now construct new examples, using Theorem[1.1. In fact, we only use the conclusion of Theorem[1.1 without existence of a $K$-rational place on $F$ (cf. the above Remark 1.4).

Theorem 2.1. Let $k$ be a field not containing a large field (e.g. $k=\mathbb{Q}$ or $k=\mathbb{F}_{p}$ ). Then there is a regular extension $K / k$ such that:

- $K$ does not contain a large field,

- for every finite split embedding problem $\mathbf{E P}_{k}$ over $k$ the lifted embedding problem $\mathbf{E P}_{K}$ has a proper regular solution over $K$; in particular, the regular inverse Galois problem is solvable over $K$,

- $K$ is hilbertian.

Proof. Let $\mathcal{E P}_{k}=\left\{\mathbf{E P}_{k}^{i} \mid i \in I\right\}$ be the set of (isomorphism classes of) all finite split embedding problems over $k$. By Theorem 1.1, we find for each $i$ a function field $F_{i} / k$ in one variable with a $k$-rational place such that $\mathbf{E P}_{F_{i}}^{i}$ has a proper regular solution over $F_{i}$. We may choose the fields $F_{i}(i \in I)$ such that each $F_{i}$ is linearly disjoint over $k$ from the compositum of all $F_{j}$ with $j \neq i$. Now let $K$ be the compositum of all $F_{i}(i \in I)$.

Then, by construction, each lifted embedding problem $\mathbf{E P}_{K}^{i}(i \in I)$ has a proper regular solution over $K$. It is also clear that $K / k$ is regular and that $K$ is hilbertian. We have to show that $K$ does not contain any large field. But this is immediate from the following lemma.

Lemma 2.2. Let $k$ be a field not containing a large field. Let $\left(F_{i}\right)_{i \in I}$ be a family of function fields over $k$, linearly disjoint over $k$ (i.e. for each $i, F_{i}$ is linearly disjoint over $k$ from the compositum of all $F_{j}$ with $i \neq j \in I$ ). Let $K=F_{I}$ be the compositum of all $F_{i}$ $(i \in I)$. Then $K$ does not contain a large field.

Proof. Assume, to the contrary, that $K$ contains a large field $L$. Then, by assumption, $L \nsubseteq k$. So we may pick some $x \in L \backslash k$. As $K / k$ is regular, $x$ is transcendental over $k$. Now let $k_{0}$ be the prime field of $k$ and choose a smooth curve $\mathcal{C}$ of genus $\geq 2$ defined over $k_{0}(x)$ with a $k_{0}(x)$-rational point $P \in \mathcal{C}\left(k_{0}(x)\right)$, but such that $\mathcal{C}$ is non-isotrivial over any constant field extension $E / k_{0}$, i.e. $\mathcal{C}$ is not birationally equivalent (over some finite extension of $E(x))$ to a curve defined over $E$ for any field $E$ containing $k_{0}$ over which $x$ remains transcendental. Such curves always exist (take e.g. a smooth model of the curve constructed in Example 2.3 below).

Now let $I_{0} \subseteq I$ be the unique minimal finite subset of $I$ such that $x \in F_{I_{0}}$, the compositum of the $F_{i}$ with $i \in I_{0}$. Let $\left\{x_{1}, \ldots, x_{n}\right\}$ be a transcendence base of $F_{I_{0}}$ over $k$ and let $E$ be the compositum of $k\left(x_{1}, \ldots, x_{n-1}\right)$ with $F_{I \backslash I_{0}}$. Then $x$ is transcendental over $E$ and $K$ is a function field in one variable over $E$. As $\mathcal{C}$ is non-isotrivial over $E$, the Mordell conjecture over function fields $([\overline{\mathrm{M}}],[\mathrm{C}]$ in characteristic $0,[\mathrm{G}]$ and $[\mathrm{S}]$ in arbitrary characteristic) implies that $\mathcal{C}(K)$ is finite. As $P \in \mathcal{C}\left(k_{0}(x)\right) \subseteq \mathcal{C}(L) \subseteq \mathcal{C}(K)$, $\mathcal{C}(L)$ is non-empty, but finite, contradicting the largeness of $L$. 
Example 2.3. Let $k$ be a field of char $k \neq 2$ and let $x$ be transcendental over $k$. Then the curve $\mathcal{C}$ defined by the equation

$$
Y^{2}=g(X):=X(X-1)(X-x)\left(X-x^{2}\right)\left(X-x^{3}\right)\left(X-x^{4}\right)
$$

is a geometrically irreducible curve of genus 2 defined over $k(x)$ with smooth $k(x)$ rational point $(0,0)$, but non-isotrivial to a curve over $E$ for any field extension $E / k$ linearly disjoint from $x$ over $k$.

If char $k=2$, one has to modify the curve replacing $Y^{2}$ by $Y^{2}-Y$.

Proof. We treat here only the case char $k \neq 2$. By [CF p. 1], $\mathcal{C}$ is a curve of genus 2 , and any curve of genus 2 over a field $F$ of characteristic $\neq 2$ is birationally equivalent over $F$ to a curve of the shape

$$
Y^{2}=g(X),
$$

where $g \in F[X]$ is separable of degree 6 , and this canonical form is unique up to a fractional linear transformation of $X$ with associated transformation of $Y$ :

$$
X \mapsto \frac{a X+b}{c X+d}, \quad Y \mapsto \frac{e Y}{(c X+d)^{3}},
$$

for some $a, b, c, d \in F$ with $a d-b c \neq 0$ and $e \in F^{\times}$.

It is clear that $(0,0)$ is a smooth $k(x)$-rational point of $\mathcal{C}$. We have to show that for any field extension $E / k$ with $x$ still transcendental over $E$ and any finite extension $F / E(x)$, the curve $\mathcal{C}$ is not birationally equivalent over $F$ to a curve defined over $E$.

Assume to the contrary that $F$ is a finite extension of $E(x)$ and that $\mathcal{C}^{\prime}$ is such a curve over $E, F$-birationally equivalent to $\mathcal{C}$. We may assume that $\mathcal{C}^{\prime}$ is in canonical form over $E$, and hence, by the remarks above, there are $a, b, c, d \in F$ with $a d-b c \neq 0$ and $e \in F^{\times}$such that the curve $\mathcal{C}^{\prime}$ is defined by

$$
(e Y)^{2}=(c X+d)^{6} \cdot g\left(\frac{a X+b}{c X+d}\right) \in E[X],
$$

where the polynomial on the right is of degree 6 in $X$. Yet this proves impossible: the polynomial on the right hand side is of the form

$$
f \cdot\left(X-\frac{b}{a}\right)\left(X-\frac{b-d}{a-c}\right)\left(X-\frac{b-d x}{a-c x}\right)\left(X-\frac{b-d x^{2}}{a-c x^{2}}\right)\left(X-\frac{b-d x^{3}}{a-c x^{3}}\right)\left(X-\frac{b-d x^{4}}{a-c x^{4}}\right),
$$

where $f=a(a-c)(a-c x)\left(a-c x^{2}\right)\left(a-c x^{3}\right)\left(a-c x^{4}\right) \in E^{\times}$. This implies that the following four elements are in the algebraic closure $\widetilde{E}$ of $E$ :

$$
\alpha:=\frac{b}{a}, \quad \beta:=\frac{b-d}{a-c}, \quad \gamma:=\frac{b-d x}{a-c x}, \quad \delta:=\frac{b-d x^{2}}{a-c x^{2}} .
$$

By elementary computations this implies a contradiction: The first two equations give

$$
b=\alpha a, \quad d=(\alpha-\beta) a+\beta c .
$$


Plugging this in the last two equations gives

$$
c=\frac{(\alpha-\beta) x+(\gamma-\alpha)}{(\gamma-\beta) x} \cdot a, \quad c=\frac{(\alpha-\beta) x^{2}+(\delta-\alpha)}{(\delta-\beta) x^{2}} \cdot a .
$$

Note that $\gamma \neq \beta$ and $\delta \neq \beta$ as $a d-b c \neq 0$. Those two expressions for $c$, however, yield the equation

$$
(\alpha-\beta)(\delta-\beta) x^{2}+(\gamma-\alpha)(\delta-\beta) x=(\alpha-\beta)(\gamma-\beta) x^{2}+(\delta-\alpha)(\gamma-\beta),
$$

which implies $\alpha=\gamma=\delta$, as $x$ is transcendental over $\widetilde{E}$. But $\alpha=\gamma$ is only possible when $a d-b c=0$ : contradiction.

Remark 2.4. Unfortunately, our construction in Theorem 2.1 does not lead to a field $K$ with the stronger property that every finite split embedding problem over $K$ has a proper regular solution over $K$ (and still $K$ not containing a large field). We do not know whether such fields exist. The problem is that iteration of our construction may well lead to a field containing a large field.

\section{The diophantine nature of the regular inverse Galois problem}

It is well known that realizability of a finite group $G$ as Galois group over a field $K$ is an elementary property of $K$, expressible by a first-order sentence in the language of rings. However, it is not a diophantine property, i.e. not expressible by an existential formula: it is, in general, not preserved when passing to a larger field. Regular realizability, in contrast, is preserved under extensions, yet we do not know whether it can be expressed by a single first-order formula. The best we can offer is an infinite disjunction of existential first-order formulas:

Theorem 3.1. For any finite group $G$ there is a sequence $(\operatorname{REG}(G, n))_{n \in \mathbb{N}}$ of first-order existential statements in the language $\{+, \cdot, 0,1\}$ of rings such that

- for all $n \in \mathbb{N}: \mathbf{R E G}(G, n) \Rightarrow \mathbf{R E G}(G, n+1)$,

- for any field $K$, the group $G$ is regularly realizable over $K$ iff $\mathbf{R E G}(G, n)$ holds in $K$ for some $n \in \mathbb{N}$.

The formulas $\mathbf{R E G}(G, n)$ can be computed effectively.

Proof. For any $n, m \in \mathbb{N}$ there is a quantifier-free formula $\operatorname{ABSIRR}\left(x_{00}, \ldots, x_{n m}\right)$ in the language of rings with free variable $\left\{x_{i j} \mid 0 \leq i \leq n, 0 \leq j \leq m\right\}$ such that for all fields $K$ and for all $a_{00}, \ldots, a_{n m} \in K$ :

$$
K \models \operatorname{ABSIRR}\left(a_{00}, \ldots, a_{n m}\right) \Leftrightarrow \underset{\text { is absolutely irreducible. }}{f(t, Y):=\sum_{i=0}^{n} \sum_{j=0}^{m} a_{i j} t^{i} Y^{j} \in K[t, Y]}
$$

This is because irreducibility of a polynomial can be expressed by a first-order formula, and by quantifier elimination over algebraically closed fields, irreducibility over the algebraic closure is equivalent to a quantifier-free formula in the language of rings with 
constants for the coefficients of the polynomial. Moreover, this quantifier-free formula can be computed effectively.

Now choose a finite group $G$ and consider an arbitrary field $K$ and some indeterminate $t$ over $K$. Then the following statements are equivalent:

(i) $G$ is regularly realizable over $K$.

(ii) There is a Galois extension $L / K(t)$ with group $G$ such that $L / K$ is a regular extension.

(iii) There is an absolutely irreducible polynomial

$$
f(t, Y):=Y^{|G|}+g_{|G|-1}(t) Y^{|G|-1}+\cdots+g_{0}(t) \in K[t, Y]
$$

such that the field $L:=\operatorname{Quot}(K[t, Y] /(f))=K(t)(Y+(f))$ is a Galois extension of $K(t)$ with group $G$.

(iv) There are polynomials $g_{i}(t) \in K[T](i=0, \ldots,|G|-1)$ and rational functions $h_{\sigma, j}(t) \in K(t)(j=0, \ldots,|G|-1, \sigma \in G)$ such that

- $f(t, Y):=Y^{|G|}+g_{|G|-1}(t) Y^{|G|-1}+\cdots+g_{0}(t) \in K[t, Y]$ is absolutely irreducible,

- for all $\sigma, \tau \in G$ :

- $f(t, Y)$ divides $f(t, \widehat{\sigma}(Y))$ in $K(t)[Y]$,

$-f(t, Y)$ divides $\widehat{\sigma \tau}(Y)-\widehat{\sigma}(\widehat{\tau}(Y))$ in $K(t)[Y]$,

$-\sigma \neq \tau \Rightarrow \widehat{\sigma}(Y) \neq \widehat{\tau}(Y)$,

$-\widehat{1}(Y)=Y$,

where for each $\sigma \in G, \widehat{\sigma}$ denotes the $K(t)$-algebra endomorphism of $K(t)[Y]$ with $\widehat{\sigma}(Y)=\sum_{j=0}^{|G|-1} h_{\sigma, j}(t) Y^{j}$.

(v) For some $n \in \mathbb{N}$ the following statement $\operatorname{REG}(G, n)$ holds in $K$ :

There are polynomials $g_{i}, h_{\sigma, j}^{ \pm}, r_{\sigma, k}^{ \pm}, s_{\sigma, \tau, l}^{ \pm} \in K[T]$ all having degree $\leq n$, where $i, j, k, l \in \mathbb{N}$ with $i, j<|G|, k, l<|G|^{2}$ and $\sigma, \tau \in G$ satisfying the conditions:

- $\operatorname{ABSIRR}$ (coefficients of $f$ ),

- for all $\sigma, \tau \in G$ :

$-f(t, Y) \cdot\left(\sum_{k=0}^{|G|^{2}-1} r_{\sigma, k}(t) Y^{k}\right)=f(t, \widehat{\sigma}(Y))$,

$-f(t, Y) \cdot\left(\sum_{l=0}^{|G|^{2}-1} s_{\sigma, \tau, l}(t) Y^{l}\right)=\widehat{\sigma \tau}(Y)-\widehat{\sigma}(\widehat{\tau}(Y))$,

$-\sigma \neq \tau \Rightarrow \widehat{\sigma}(Y) \neq \widehat{\tau}(Y)$,

$-\widehat{1}(Y)=Y$,

where $f$ and $\widehat{\sigma}$ are as in (iv) and where $p=p^{+} / p^{-} \in K(t)$ for any pair of polynomials $p^{+}, p^{-} \in K[t]$.

Note that in (ii), $f$ being absolutely irreducible implies $L / K$ being regular, and that the $g_{i}$ may be taken to be polynomials rather than rational functions (multiply $f$ by the $|G|$-th power of the product $d(t)$ of the denominators of the $g_{i}$ and replace $Y$ by $\left.d(t) \cdot Y\right)$.

In (iv), the four conditions listed for $\sigma, \tau \in G$ say that $\widehat{\sigma}(Y)+(f)$ is a zero of $f$ in $L:=K(t)(Y+(f))$ inducing some $\widetilde{\sigma} \in \operatorname{Aut}(L / K(t))$ with $\widetilde{\sigma \tau}=\widetilde{\sigma} \circ \widetilde{\tau}, \widetilde{\sigma} \neq \widetilde{\tau}$ for 
$\sigma \neq \tau$ and $\tilde{1}=\operatorname{id}_{L}$. So

$$
G \rightarrow \operatorname{Aut}(L / K(t)), \quad \sigma \mapsto \widetilde{\sigma},
$$

is an injective group homomorphism, and as $\operatorname{deg}_{Y} f=|G|, L / K(t)$ is Galois and the homomorphism is also onto.

Obviously, REG $(G, n)$ can be expressed as an existential statement in the language of rings as ABSIRR was a quantifier-free formula and all the polynomial equations resp. inequalities are equivalent to a conjunction resp. disjunction of equations resp. inequalities of the corresponding coefficients.

It is clear that this procedure is effective since ABSIRR was obtained in an effective way. And, finally, $\mathbf{R E G}(G, n)$ gets weaker with increasing $n$.

We do not know whether the $n$ in the theorem can be uniformly bounded, i.e. whether, given a finite group $G$, there is a natural number $n=n(G)$ such that for any $m \in \mathbb{N}$ and any field $K$,

$$
K \models \mathbf{R E G}(G, m) \Rightarrow K \models \mathbf{R E G}(G, n) .
$$

If there is such a uniform bound, then the question whether or not $G$ is regularly realizable over a given field $K$ is an existential first-order question. It is effectively decidable provided that $K$ has a decidable existential theory. This is, of course, the case e.g. if $K$ is finite, but it is not known, e.g., when $K=\mathbb{Q}$.

If, on the other hand, there is no such uniform bound then for any $m \in \mathbb{N}$, we find a field $K_{m}$ with $K_{m} \models \neg \mathbf{R E G}(G, m)$. An ultraproduct of these $K_{m}$ then gives a field $K$ over which $G$ is not regularly realizable.

Remark 3.2. The formula $\operatorname{REG}(G, n)$ says nothing explicit about ramification data. Yet, implicitly, it does: if $K \models \mathbf{R E G}(G, n)$ then $G$ can be realized as the Galois group of an extension $L / K(t)$, regular over $K$ and ramified in at most $2 n \sharp G+1$ points.

Proof. This is because ramification only occurs at infinity or at points corresponding to the divisors of the discriminant of $F$ which is a polynomial of degree $\leq 2 n \sharp G$ in $t$.

As an illustration of this model-theoretic view on the regular inverse Galois problem (RIGP) let us prove the following slight generalization of the RIGP-part of the HarbaterPop theorem (every finite group being regularly realizable over a large field). Again, this is certainly known to the experts (cf. [DD, Theorem 4.2] for a variant with varieties in place of curves).

Observation 3.3. Let $K$ be a field such that every smooth curve $\mathcal{C}$ defined over the prime field $k$ of $K$ (so $k=\mathbb{Q}$ or $k=\mathbb{F}_{p}$ ) and having a $k$-rational point has infinitely many $K$-rational points. Then the regular inverse Galois problem is solvable over $K$.

Proof. Choose an arbitrary finite group $G$. By Theorem 1.1 , there is a function field $F / k$ in one variable with a $k$-rational place such that $G$ is regularly realizable over $F$. So, by Theorem 3.1, $F \models \mathbf{R E G}(G, n)$ for some $n \in \mathbb{N}$. Let $\mathcal{C}$ be a smooth curve over $k$ with function field $F=k(\mathcal{C})$. Then $\mathcal{C}(k) \neq \emptyset$, and hence, by our hypothesis on $K$, 
$|\mathcal{C}(K)|=\infty$. Therefore, any saturated elementary extension $K^{\star} \succ K$ contains the coordinates of a point on $\mathcal{C}$ which is generic over $K$ and so also over $k$. This means that $K^{\star}$ contains a copy of $F$. Thus we have the implications

$$
F \models \mathbf{R E G}(G, n) \Rightarrow K^{\star} \models \mathbf{R E G}(G, n) \Rightarrow K \models \mathbf{R E G}(G, n) .
$$

Applying Theorem 3.1 once more, we conclude that $G$ is regularly realizable over $K$. Since $G$ was chosen arbitrarily, the observation is proved.

We do not know whether the field $K$ constructed in Theorem 2.1 satisfies the hypothesis of the above observation. Yet, if one adjoins to the prime field $k$ for each curve $\mathcal{C}$ over $k$ with $\mathcal{C}(k) \neq \varnothing$ infinitely many independent generic points one obtains a field $K$ satisfying the hypothesis of the observation, and again by Lemma 2.2, $K$ does not contain a large field.

\section{Rationality conditions}

It should be mentioned that throughout the paper regular realizability of a finite group $G$ over a field $K$ may also be replaced by the stronger condition that $G$ be realizable as the Galois group of a Galois extension $L / K(t)$ with $L / K$ regular and $L$ having a $K$-rational place. This is because the theorem of Harbater-Pop in fact proves this stronger property for large $K$ and because the extra condition is also expressible by an existential formula: just add to the formula $\operatorname{REG}(G, n)$ in Theorem 3.1 the formula ' $\exists x, y f(x, y)=0$ '. From this it is clear that one obtains the following stronger version of Corollary 2 from [FV]:

Corollary 3.4. Let $G$ be a finite group. Then for almost all finite fields $k, G$ is regularly realizable over $k$ as the Galois group of an extension $L / k(t)$ with a $k$-rational place on $L$.

Acknowledgements. I would like to thank Pierre Dèbes for encouraging me to write down this note.

\section{References}

[C] Coleman, R. F.: Manin's proof of the Mordell conjecture over function fields. Enseign. Math. 36, 393-427 (1990) Zbl 0729.14018 MR 92e:11069

[CF] Cassels, J. W. S., Flynn, E. V.: Prolegomena to a Middlebrow Arithmetic of Curves of Genus 2, London Math. Soc. Lecture Note Ser. 230, Cambridge Univ. Press (1996) Zbl 0857.14018 MR 97i:11071

[CT] Colliot-Thélène, J.-L.: Rational connectedness and Galois covers of the projective line. Ann. of Math. 151, 359-373 (2000) Zbl 0990.12003 MR 2001b:14046

[DD] Dèbes, P., Deschamps, B.: The regular inverse Galois problem over large fields. In: L. Schneps and P. Lochak (eds.), Geometric Galois Actions II, London Math. Soc. Lecture Note Ser. 243, Cambridge Univ. Press, 119-138 (1997) Zbl 0905.12004 MR 99j:12002

[FV] Fried, M. D., Völklein, H.: The inverse Galois problem and rational points on moduli spaces. Math. Ann. 290, 771-800 (1991) Zbl 0763.12004 MR 93a:12004

[G] Grauert, H.: Mordells Vermutung über rationale Punkte auf algebraischen Kurven und Funktionenkörper. Publ. Math. IHES 25, 131-149 (1965) Zbl 0137.40503 MR 36 \#5139 
[HJ] Haran, D., Jarden, M.: Regular split embedding problems over complete valued fields. Forum Math. 10, 329-351 (1998) Zbl 0903.12003 MR 99e:12007

$[\mathrm{H}]$ Harbater, D.: Galois coverings of the arithmetic line. In: Number Theory, Lecture Notes in Math. 1240, Springer, 165-195 (1987) Zbl 0627.12015 MR 88h:14020

[M] Manin, Yu. I.: Rational points of algebraic curves over function fields. Izv. Akad. Nauk SSSR 27, 1395-1440 (1963) (in Russian); English transl.: AMS Transl. Ser. II 50, 189-234 (1966) Zbl 0178.55102

[P] Pop, F.: Embedding problems over large fields. Ann. of Math. 144, 1-34 (1996) Zbl 0862.12003 MR 97h:12013

[S] Samuel, P.: Complément à un article de Grauert sur la conjecture de Mordell. Publ. Math. IHES 29, 55-62 (1966) Zbl 0144.20102 MR 34 \#4272 